\title{
One-Dimensional Aromatic Crystals in Solution VI. Synthesis and Spectroscopic Characterization of Poly( $\beta$-9-anthrylmethyl L-aspartate)
}

\author{
Masahiko Sisido, Akira OKamoto, ${ }^{*}$ Syun EgusA, * \\ and Yukio IMANISHI* \\ Research Center for Medical Polymers and Biomaterials, Kyoto University, \\ Sakyo, Kyoto 606, Japan \\ *Department of Polymer Chemistry, Kyoto University, \\ Sakyo, Kyoto 606, Japan
}

(Received June 21, 1985)

\begin{abstract}
An aromatic poly( $\alpha$-amino acid) carrying anthryl chromophores, poly(9anthrylmethyl L-aspartate), was first synthesized in a form of a block copolymer with poly( $\gamma$-benzyl DL-glutamate) which functions as a solubilizing component. The polypeptide in trimethyl phosphate solution showed a marked exciton splitting in the circular dichroism at the ${ }^{1} \mathrm{~B}_{\mathrm{b}}$ absorption band, indicating a helical arrangement of anthryl groups along the helical main chain. Electronic interactions in the ground and excited states of the aromatic polypeptide were studied by absorption, fluorescence, circular dichroism (CD), circularly polarized fluorescence (CPF), and fluorescence-detected circular dichroism (FDCD) spectroscopy. In the ground state, some portion of the anthryl groups was found to form dimers or higher aggregates. The fluorescence spectra of the polymer consisted of monomer fluorescence with a small contribution of the dimer excited state. The CPF spectrum indicated that the monomer excited state partially delocalizes over neighboring anthryl groups. The dimer emission also showed a significant circular polarization, indicating dissymmetric structure of the dimer. The FDCD spectrum, when monitored by the dimer fluorescence, showed a more intense $C D$ signal than that of conventional absorption $C D$, indicating that the dimer is formed in a helical part of the polypeptide. On the basis of these observations, a scheme was proposed for the electronic states of the anthryl polypeptide.
\end{abstract}

KEY WORDS Poly(9-anthrylmethyl L-aspartate) / Circular Dichroism /

Fluorescence / Circularly Polarized Fluorescence / Fluorescence-Detected Circular Dichroism /

Electric and electronic properties of polypeptides are of potential applicability, but have been attracting little interest as compared with biological properties. Helix structure of polypeptides is one of rare examples of one-dimensional structure which is maintained in solution. The strong anisotropy and dissymmetry of the helix may induce a variety of unique electric and electronic properties. Appropriate utilizations of these unique properties will open a new field of polypeptide chemistry. In the case of piezoelectricity, for example, a unidirectional ar- rangement of dipolar amide groups of polypeptide chain has been shown to induce a macroscopic polarization. ${ }^{1,2}$ The piezoelectricity may be much enhanced if more polar groups are attached to side chains of a polypeptide. Polypeptides are also promissing as nonlinear optical materials for second-harmonic generation (SHG) and so on, because of their anisotropic and dissymmetric structure. ${ }^{3}$ The nonlinear optical effects will be enhanced if more polarizable chromophores are attached to side chains of a polypeptide.

Potentiality of chromophoric polypeptides 
for the application to molecular electronic devices has been proposed. ${ }^{4}$ The regular and rigid framework of the helix will realize definite spatial arrangement of chromophores which function as electron sources, sinks, conductors, etc.

The present authors have reported the synthesis and conformational analysis of aromatic polypeptides of the form I, carrying 1-naphthyl, ${ }^{5}$ 2-naphthyl, ${ }^{6}$ and 1-pyrenyl chromophores. $^{7-9}$<smiles>N#CC(CBr)C(=O)[O-]</smiles>

The poly(arylalanine)s were shown to take helical conformations in solution along which the aryl chromophores are helically arranged. Although poly(arylalanine)s are ideal polymers to attain the helical arrangement of chromophores, the synthesis and optical resolution of aromatic amino acids (arylalanines), which are not present in nature, are often extremely difficult and the polymerization of the corresponding amino acid $\mathrm{N}$ carboxyanhydrides sometimes fails to proceed.

The synthesis of aromatic polypeptides by the attachment of chromophores to the side chains of poly(aspartate)s, ${ }^{10,11}$ poly(glutamate)s, ${ }^{10,12}$ and poly(lysine)s, ${ }^{13,14}$ is a simpler and more general way. However, in these polypeptides the side-chain chromophores are linked to the main chain by long spacer groups, i.e., $-\mathrm{CH}_{2}-\mathrm{COO}-$ for aspartates, $-\left(\mathrm{CH}_{2}\right)_{2}-\mathrm{COO}-$ for glutamates, and $-\left(\mathrm{CH}_{2}\right)_{4}$ -NHCO- for lysines. No evidence has been reported for the helical arrangement of the side-chain chromophores in these aromatic polypeptides.

The strategy employed in the present research is to link a bulky chromophore to poly(aspartate) by an ester linkage. Although the spacer group in this case is relatively long, steric interactions between bulky side-chain chromophores may enforce them to arrange helically along the main chain. In this report, the synthesis and spectroscopic investigation of poly(9-anthrylmethyl L-aspartate) (II) are described.<smiles>CNC(CC(=O)OCc1ccccc1)C(=O)OC</smiles>

\section{EXPERIMENTAL}

\section{Materials}

The attachment of 9-anthrylmethyl group to the side chain of L-aspartate was first attempted by a direct esterification of L-aspartic acid with 9-anthrylmethanol in dioxane containing $p$-toluenesulfonic acid. The reaction, however, was very slow and a prolonged refluxing yielded a deeply colored material. Therefore, the route shown in Scheme I was employed.
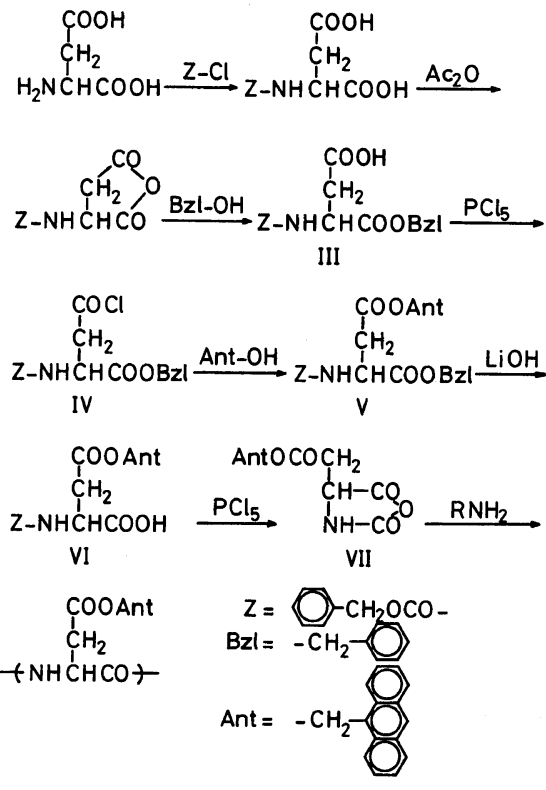

Scheme I 
No racemization was found to occur during the reaction. The aromatic amino acid was converted to $\mathrm{N}$-carboxyanhydride (NCA) derivative and polymerized with an initiator having primary amino group. Since the homopolymer of 9-anthrylmethyl L-aspartate was insoluble in organic solvents, a preformed poly( $\gamma$-benzyl DL-glutamate) was used as an initiator for the NCA polymerization to prepare a block copolymer soluble in organic solvents.

$\alpha$-Benzyl $\quad N$-benzyloxycarbonyl-L-aspartate $\beta$-chloride (IV)

$\alpha$-Benzyl $N$-benzyloxycarbonyl-L-aspartate (III) prepared in our laboratory was dissolved in anhydrous ether at $0^{\circ} \mathrm{C}$ and about 5-fold excess of phosphorous pentachloride was added. After vigorous shaking for $30 \mathrm{~min}$ at $0^{\circ} \mathrm{C}$, the solution was filtered and dry hexane was added to precipitate the acid chloride. The crystal was washed with hexane and dried in vacuum. Infrared spectrum showed a carbonyl stretching peak at $1792 \mathrm{~cm}^{-1}$ which is characteristic of acid chloride.

$\alpha$-Benzyl $\beta$-9-Anthrylmethyl $N$-Benzyloxycarbonyl-L-aspartate $(V)$

The acid chloride (IV) ( $1 \mathrm{~g}$ ) was dissolved in anhydrous dioxane $(10 \mathrm{ml})$ and an equimolar amount of 9-anthrylmethanol (Aldrich) $(0.55 \mathrm{~g})$ and a 2-fold excess of pyridine $(0.43 \mathrm{ml})$ were added. The mixture was stirred at room temperature overnight and the salt precipitated was filtered off. The solvent was evaporated and the residue was redissolved in ether. The etheral solution was washed with water and dried with anhydrous sodium sulfate. The ether was evaporated and the residual crude crystal was recrystallized from a mixed solvent of methanol/ether/hexane to give a yellow crystal. Yield $0.94 \mathrm{~g}(65 \%) \mathrm{mp}$ 115-115.5 $5^{\circ}$. Anal. Calcd for $\mathrm{C}_{34} \mathrm{H}_{29} \mathrm{O}_{6} \mathrm{~N}: \mathrm{C}$, $74.57 \%$; H, 5.34\%; N, 2.56\%. Found: C, $74.66 \%$; H, 5.26\%; N, $2.54 \%$.

$\beta$-9-Anthrylmethyl N-Benzyloxycarbonyl-Laspartate (VI)

The diester (V) $(0.5 \mathrm{~g})$ was dissolved in ace- tone $(10 \mathrm{ml})$ and 1.1 molar equivalent of $0.4 N$ aqueous $\mathrm{LiOH}$ solution was added dropwise over $1 \mathrm{~h}$. The solution was further stirred for $1 \mathrm{~h}$ and the acetone was removed. The residual solid was washed with ether three times and dissolved in ethyl acetate/water mixture. Dilute hydrochloric acid was then added until the solid disappeared. The organic layer was washed with water four times and dried with anhydrous sodium sulfate. The ethyl acetate was evaporated and the residual crude crystal was recrystallized from ether/hexane mixed solvent. ${ }^{1} \mathrm{H}$ NMR spectrum in $\mathrm{CDCl}_{3}$ showed a single peak in the benzyl methylene region. Yield $0.2 \mathrm{~g}(48 \%), \mathrm{mp} 138.5-139.5^{\circ} \mathrm{C} .[\alpha]_{\mathrm{D}}^{29^{\circ}}=$ $6.24\left(c, 25 \mathrm{gl}^{-1}, \mathrm{CHCl}_{3}\right)$. Anal. Calcd for $\mathrm{C}_{27} \mathrm{H}_{23} \mathrm{O}_{6} \mathrm{~N}: \mathrm{C}, 70.89 \% ; \mathrm{H}, 5.06 \%$; N, 3.06\%. Found: C, $70.90 \%$; H, $4.97 \%$; N, $2.94 \%$.

Test for the Absence of Racemization

The extent of racemization during the above steps was examined as follows. The monoester (VI) and an equimolar mixture of 9-anthrylmethanol and $N$-benzyloxycarbonyl-L-aspartic acid were dissolved in acetone separately. To each solution was added a 3-fold excess of $3 \mathrm{~N}$ aqueous $\mathrm{NaOH}$ solution. The change in optical rotation of each solution was followed at $420 \mathrm{~nm}$. After one day, the $[\alpha]_{420}$ values of the two solution coincided within the experimental error $\left(60 \pm 1^{\circ}\right)$. The product from the monoester by this treatment was confirmed to be the original $N$-benzyloxycarbonyl-L-aspartic acid by IR spectroscopy. Thus the absence of racemization during the synthesis of the monoester (VI) was confirmed.

9-Anthrylmethyl L-aspartate $N$-carboxyanhydride (NCA) (VII)

The monoester (VI) (1 g) was dissolved in anhydrous tetrahydrofuran $(20 \mathrm{ml})$ and cooled at $0^{\circ} \mathrm{C}$. Phosphorous pentachloride $(0.7 \mathrm{~g})$ was then added and stirred for 30 min under cooling in an ice bath. Addition of dry hexane gave a precipitate, which was recrystallized from ethyl acetate/hexane mixed solvent to give a slightly yellowish crystal. Yield $0.2 \mathrm{~g}(20 \%)$, $\mathrm{mp} 147-149^{\circ} \mathrm{C}$. Infrared spectrum showed a 
pair of carbonyl stretching peaks (1858, $\left.1782 \mathrm{~cm}^{-1}\right)$ characteristic of the NCA ring.

Poly( $\beta$-9-anthrylmethyl L-aspartate- $b-\gamma$-benzyl DL-glutamate) (VIII)

$\gamma$-Benzyl DL-glutamate NCA was polymerized in dimethylformamide (DMF) with $n$ hexylamine as an initiator at room temperature. The number average degree of polymerization was adjusted to 120 by the NCA/amine ratio. Immediately after the disappearance of the IR absorption characteristic of the NCA (1 day), the polymer solution was used to initiate the polymerization of 9-anthrylmethyl Lasparate $\mathrm{NCA}$ in $\mathrm{DMF} \quad([\mathrm{NCA}]=7.4 \times$ $10^{-2} \mathrm{M}$ ) at room temperature. The NCA/ amine ratio of the second polymerization was 47. The IR absorption of the latter NCA disappeared after five days. The polymerization mixture was diluted with DMF to an appropriate concentration and the absorption coefficient of the anthryl groups of the polymer chain was determined $\left(\varepsilon_{367}=4.2 \times 10^{3}\right)$, assuming a $100 \%$ conversion of the latter NCA. The absorption coefficient was used to determine the polymer concentration for spectroscopic measurements.

A major portion of the polymer solution was poured into ether and the precipitate obtained was washed with ether and fractionated by gel chromatography (Mercko gel OR-PVA 80000 in DMF). The block copolymer showed a bimodal elution diagram ranging from the elution limit of the gel to low molecular weight region $(M=80000-1000)$, whereas the poly $(\gamma$-benzyl DL-glutamate) used as the initiator showed a single elution peak at $M=20000$. For the following spectroscopic study, higher molecular weight fraction than $M=40000$ was used. Since the poly $(\gamma$-benzyl DL-glutamate) unit does not affect any spectroscopic properties of $\operatorname{poly}(\beta$-9-anthrylmethyl L-aspartate) unit, except for a small contribution in absorption spectrum, the block copolymer will be referred simply as $\operatorname{poly}(\beta-9$ anthrylmethyl L-aspartate $)$ or poly $\left[\mathrm{Asp}\left(\mathrm{OCH}_{2}\right.\right.$ 9-Ant)], hereafter.

\section{Measurements}

Spectroscopic measurements were performed mostly in trimethyl phosphate (TMP) solution. The latter solvent was distilled before each measurement. Following spectrometers were used: Hitachi EPS-3T (Uv-vis absorption), Hitachi MPF-4 (Fluorescence), Jasco J-20 (CD), Jasco FCD-1F (CPF). ${ }^{15}$ Fluorescence-detected circular dichroism was measured on a Jasco J-20 machine equipped with a cutoff filter for the excitation light (saturated $\mathrm{NaNO}_{2}$ solution in a $1 \mathrm{~cm}$ cell and appropriate glass filters), a photomultiplier tube (Hamamatsu R-268), and a preamplifier. ${ }^{8,16,17}$ The output from the preamplifer was connected to the CD amplifier of the J-20 machine and the recorder output from the J-20 machine was put into a microcomputer. Typically, 4 to 8 runs were averaged to obtain an FDCD spectrum.

\section{RESULTS AND DISCUSSION}

\section{Absorption Spectrum and Circular Dichroism}

Figure 1 shows absorption and CD spectra of poly[Asp $\left.\left(\mathrm{OCH}_{2}-9-\mathrm{Ant}\right)\right]$ and the monomeric diester (V) in TMP. A typical exciton couplet is observed at the ${ }^{1} B_{b}$ absorption band $(250 \mathrm{~nm})$ of the anthryl polypeptide, indicating that the anthryl groups are regularly arranged along a helical polypeptide chain and interact with each other at the Frank-Condon state of the ${ }^{1} B_{b}$ excited state. The exciton couplet may be the first example observed in a series of polypeptides carrying side-chain chromophores across spacers with more than one methylene unit. Thus it was concluded that though side-chain chromophores are linked to the polypeptide chain by a relatively long and flexible chain $\left(-\mathrm{CH}_{2}-\mathrm{CO}-\mathrm{O}-\mathrm{CH}_{2}-\right.$ in the present case), bulky chromophores arrange themselves so as to minimize the repulsive interactions.

A moderately large positive $\mathrm{CD}$ is seen at $408 \mathrm{~nm}$ and a shoulder is evident around 400$420 \mathrm{~nm}$ in the absorption spectrum of 


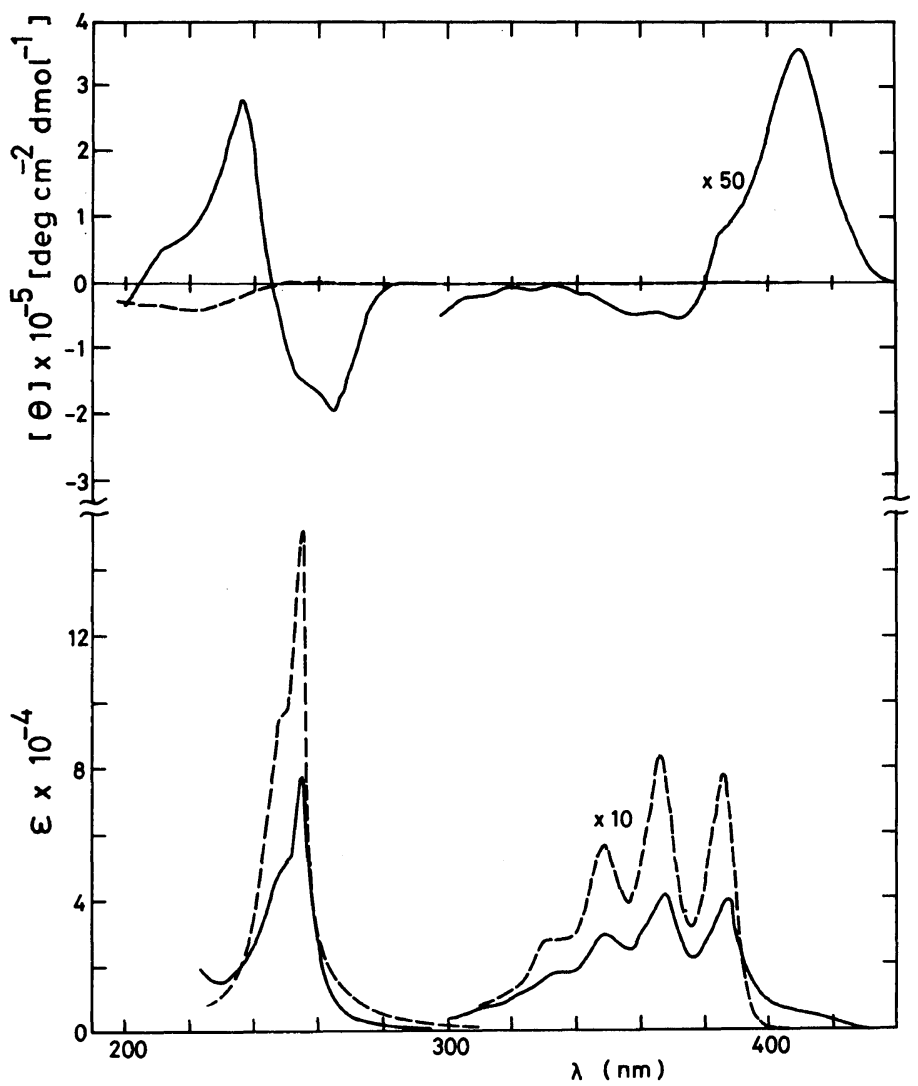

Figure 1. Circular dichroism (top) and absorption (bottom) spectra of poly[Asp( $\left.\left.\mathrm{OCH}_{2}-9-\mathrm{Ant}\right)\right](-)$ and $\mathrm{Z}$-Asp $\left(\mathrm{OCH}_{2}-9-\mathrm{Ant}\right)-\mathrm{OBzl}(----)$ in TMP. [Asp] $=1.0 \times 10^{-4} \mathrm{~mol} \mathrm{l}^{-1}$, room temperature.

poly[Asp $\left.\left(\mathrm{OCH}_{2}-9-\mathrm{Ant}\right)\right]$. The latter wavelength is too high to be interpreted as an exciton formation in the ${ }^{1} \mathrm{~L}_{\mathrm{a}}$ absoption band. As has been reported on polyesters carrying anthryl groups ${ }^{18}$ this band may be assignable to the dimer or higher aggregate in the ground state of anthryl groups. No dimer or higher aggregate formation is detected in the monomeric model compound.

As a whole, absorption intensity of poly[Asp $\left.\left(\mathrm{OCH}_{2}-9-\mathrm{Ant}\right)\right]$ is much weaker than that of the model compound. The hypochromicity also suggests interactions in the FrankCondon state of anthryl groups.
Fluorescence and Fluorescence Excitation Spectra

Figure 2 shows fluorescence spectra of poly[Asp $\left.\left(\mathrm{OCH}_{2}-9-\mathrm{Ant}\right)\right]$ and the model compound with different excitation wavelengths. The spectrum of the polymer excited at shorter wavelengths consists mostly of monomer fluorescence having the same peak positions as those of the model compound. The spectrum, however, contains longer wavelength component than the monomer fluorescence, which may be assigned to fluorescence from excimer and/or excited dimer or higher aggregates. The fluorescence spectrum when excited at $410 \mathrm{~nm}$ should represent that of the dimer or higher aggregates, since monomeric anthracene does not absorb at this excitation wavelength. The 


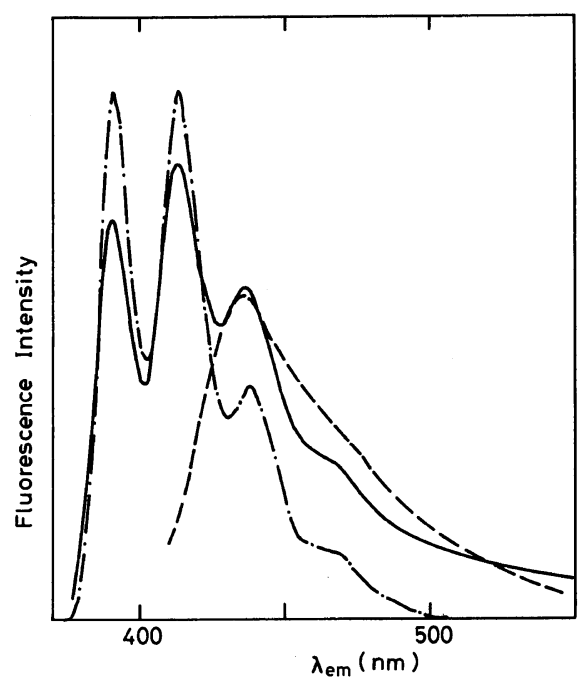

Figure 2. Fluorescence spectra of poly[ $\mathrm{Asp}\left(\mathrm{OCH}_{2}-9\right.$ Ant)] excited at $367 \mathrm{~nm}(-)$ and at $410 \mathrm{~nm}(-----)$ and of $\mathrm{Z}$-Asp $\left(\mathrm{OCH}_{2}-9-\mathrm{Ant}\right)-\mathrm{OBzl}$ excited at $367 \mathrm{~nm}$ (- - ). [Ant] $=1.0 \times 10^{-5} \mathrm{moll}^{-1}$ in TMP, room temperature.

fluorescence spectrum of the excited dimer or aggregates is similar to the longer wavelength component of the spectrum which was obtained by the excitation at $367 \mathrm{~nm}$.

Figure 3 shows fluorescence excitation spectra of the polymer and the model compound monitored at 413 and $500 \mathrm{~nm}$. The spectrum of the polymer monitored at the monomer fluorescence $\left(\lambda_{\mathrm{em}}=413 \mathrm{~nm}\right)$ is almost identical to that of the model compound, indicating the absence of strong ground state interactions between anthryl groups, except for the dimer or higher aggregate formation. The spectrum of the polymer monitored at $\lambda_{\mathrm{em}}=500 \mathrm{~nm}$ should correspond to the absorption spectrum of dimer or higher aggregates superposed on the absorption spectrum of the monomeric anthryl groups. The ratio of the two absorption spectra should depend on the extent of energy transfer from the monomer excited state to the dimer or higher aggregates or to the excimer. Indeed, the excitation spectrum appears to be a mixture of a broad structureless spectrum and the monomeric spectrum of anthracene,

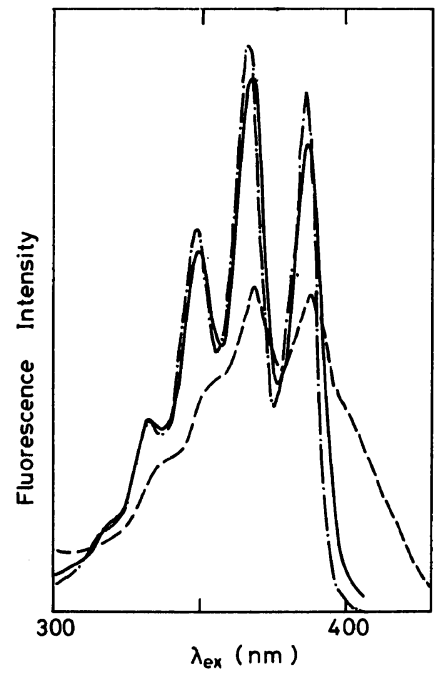

Figure 3. Fluorescence excitation spectra of poly[Asp $\left.\left(\mathrm{OCH}_{2}-9-\mathrm{Ant}\right)\right]$ monitored at $413 \mathrm{~nm}(-)$ and at $500 \mathrm{~nm}$ (-----) and of $\mathrm{Z}-\mathrm{Asp}\left(\mathrm{OCH}_{2}-9-\mathrm{Ant}\right)-\mathrm{OBzl}$ monitored at $413 \mathrm{~nm}(---)$. [Ant] $=1.0 \times 10^{-5} \mathrm{moll}^{-1}$ in TMP, room temperature.

indicating the presence of energy transfer process from the monomer excited state to the dimer or aggregates.

The energy transfer process is also evident from the study of fluorescence polarization of the polymer. The largest polarization was $P=$ 0.03 for $\lambda_{\mathrm{ex}}=387 \mathrm{~nm}$ and $\lambda_{\mathrm{em}}=480 \mathrm{~nm}$ at $15.5^{\circ} \mathrm{C}$ and the polarization decreased with increasing temperature. It was 0.02 when the monitor wavelength was $413 \mathrm{~nm}$ at $15.5^{\circ} \mathrm{C}$. The virtual depolarization indicates that the energy absorbed by an anthryl group migrates among the side-chain chromophores.

\section{Circularly Polarized Fluorescence}

CPF spectroscopy measures the difference in intensities of left- $\left(I_{\mathrm{L}}^{\mathrm{em}}\right)$ and right- $\left(I_{\mathrm{R}}^{\mathrm{em}}\right)$ circularly polarized components in fluorescence emitted from a chiral fluorophore which was excited by unpolarized light. ${ }^{17,19}$ The CPF spectrum provides information on the chiral geometry and interactions in the excited state of the fluorophore. CPF spectrum is usually represented by the Kuhn's emission dissym- 


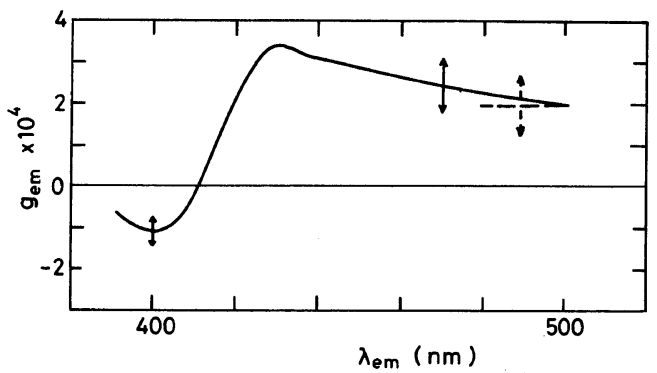

Figure 4. Circularly polarized fluorescence spectra of poly[Asp $\left.\left(\mathrm{OCH}_{2}-9-\mathrm{Ant}\right)\right]$ excited at $320 \mathrm{~nm}$ (-) and at $410 \mathrm{~nm}$ (-----). [Ant] $=2.7 \times 10^{-4} \mathrm{moll}^{-1}$ in TMP, room temperature. The vertical bars represent noise levels. Measuring conditions: time constant $=12.5 \mathrm{~s}$, scan speed $=1.5 \mathrm{~nm} \mathrm{~min}-1$, slit width $= \pm 6 \mathrm{~nm}$, cell length $=$ $1 \mathrm{~cm}$.

metry factor $g_{\mathrm{em}}$ as a function of the emission wavelength.

$$
P_{\mathrm{em}}=2\left(I_{\mathrm{L}}^{\mathrm{em}}-I_{\mathrm{R}}^{\mathrm{em}}\right) /\left(I_{\mathrm{L}}^{\mathrm{em}}+I_{\mathrm{R}}^{\mathrm{em}}\right)
$$

Figure 4 shows CPF spectra of poly[Asp $\left(\mathrm{OCH}_{2}\right.$-9-Ant $\left.)\right]$ in TMP at different excitation wavelengths. The spectrum with $\lambda_{\mathrm{ex}}=320 \mathrm{~nm}$ shows negative $g_{\mathrm{em}}$ around $400 \mathrm{~nm}$, indicating that the monomer excited state of anthracene in the polymer interacts with neighboring ground state chromophores to form an exciton state. The exciton emission is the first example observed in a series of aromatic polypeptides, including poly(L-1- and 2-naphthylalanines) ${ }^{15}$ and poly(L-1-pyrenylalanine). ${ }^{8}$ The lowest excited state of anthracene $\left({ }^{1} \mathrm{~L}_{\mathrm{a}}\right)$ has a moderately large transition dipole moment, whereas that of naphthalene or pyrene has little transition dipole. Hence, the observation of the exciton state only in poly[Asp $\left.\left(\mathrm{OCH}_{2}-9-\mathrm{Ant}\right)\right]$ is accounted for by a strong dipole-dipole interaction between excited- and ground-stage anthryl chromophores. However, the extent of delocalization of the excitation energy in poly[Asp $\left.\left(\mathrm{OCH}_{2}-9-\mathrm{Ant}\right)\right]$ may be very small, as has been suggested by the coincidence of the peak positions in the fluorescence spectra of the polymer and the model compound (Figure 2).
Positive CPF signal is observed at longer emission wavelengths than $420 \mathrm{~nm}$, indicating that the excimer and/or dimer (or higher aggregates) has a strongly dissymmetric geometry. Presumably it is formed in a helical region of the polymer by a local deformation of the side chain.

When the polymer was excited at the absorption band of the dimer or aggregates $\left(\lambda_{\mathrm{ex}}=410 \mathrm{~nm}\right)$, the CPF signal at 480 $500 \mathrm{~nm}$ was almost the same as that of $\lambda_{\mathrm{ex}}=$ $320 \mathrm{~nm}$. This suggests either that the excited dimer has geometrical and electronic structures similar to the excimer or that no excimer exists on the polymer chain. The latter idea seems more probable, since it is unlikely that the dimer excited state, which will relax to the ground state dimer, has the same geometrical and electronic structures as the excimer which will relax to a ground state monomer.

\section{Fluorescence-Detected Circular Dichroism}

FDCD spectroscopy measures the difference in fluorescence intensities when a chiral sample is excited by left- $\left(I_{\mathrm{L}}^{\mathbf{e x}}\right)$ and rihgt- $\left(I_{\mathrm{R}}^{\mathbf{e x}}\right)$ circularly polarized lights. ${ }^{16,17}$ When the sample solution contains a single non-interacting chiral fluorophore, the FDCD should afford the same information as the conventional absorption CD. However, if the sample consists of several kinds of chiral species which fluoresce at different wavelengths with different quantum yields, the FDCD monitored at a particular wavelength corresponds to the CD of the species which fluoresces at that wavelength. Therefore, the FDCD spectroscopy is a powerful technique to measure $C D$ spectrum of a particular component selectively from a mixture of several fluorophores. FDCD spectra are usually presented by the Kuhn's excitation dissymmetry factor $g_{\mathrm{ex}}$, as a function of the excitation wavelength.

$$
g_{\mathrm{ex}}=2\left(I_{\mathrm{L}}^{\mathrm{ex}}-I_{\mathrm{R}}^{\mathrm{ex}}\right) /\left(I_{\mathrm{L}}^{\mathrm{ex}}+I_{\mathrm{R}}^{\mathrm{ex}}\right)
$$

The FDCD spectrum is compared with ab- 


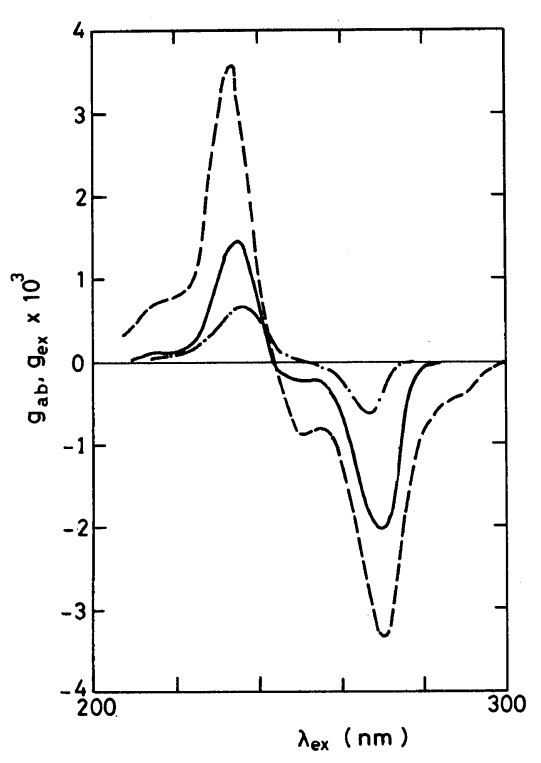

Figure 5. Fluorescence-detected circular dichroism of poly[Asp $\left.\left(\mathrm{OCH}_{2}-9-\mathrm{Ant}\right)\right]$ monitored at longer wavelengths than $500 \mathrm{~nm}(-----)$, and at $400-450 \mathrm{~nm}$ $\left(--\right.$ ). $[$ Ant $]=2.0 \times 10^{-5} \mathrm{moll}^{-1}$ in TMP at room temperature. Absorption circular dichroism of poly$\left[\mathrm{Asp}\left(\mathrm{OCH}_{2}-9-\mathrm{Ant}\right)\right]$ in the form of the dissymmetry factor is also shown (-). Measuring conditions for the FDCD spectra: time constant $=16 \mathrm{~s}$, scan speed $=$ $2 \mathrm{~nm} \min ^{-1}$, cell length $=1 \mathrm{~cm}$, cutoff filter $=\mathrm{NaNO}_{2}$ saturated solution + appropriate glass filters.

sorption $\mathrm{CD}$ which is presented in a form of the absorption dissymmetry factor $g_{\mathrm{ab}}$.

$$
g_{\mathrm{ab}}=2\left(\varepsilon_{\mathrm{L}}-\varepsilon_{\mathrm{R}}\right) /\left(\varepsilon_{\mathrm{L}}+\varepsilon_{\mathrm{R}}\right)
$$

To calculate $g_{\mathrm{ab}}$, the difference of the absorption coefficients obtained as an recorder output of $C D$ spectrometer was divided by the absorption spectrum measured using the same optical system as the CD spectrometer.

Figure 5 compares an FDCD spectrum of poly[Asp $\left.\left(\mathrm{OCH}_{2}-9-\mathrm{Ant}\right)\right]$ in TMP solution monitored at the dimer and/or excimer fluorescence wavelengths $\left(\lambda_{\text {ex }}>500 \mathrm{~nm}\right)$ and an absorption CD in the form of $g_{\mathrm{ab}}$. Interestingly, the $g_{\mathrm{ex}}$ is larger than $g_{\mathrm{ab}}$, indicating that the excitation energy of the dimer excited state is mostly originated from the monomer excited states which are in a helical part of the polymer. Therefore, it is concluded that the dimer is formed on the helical part of the polymer, possibly by a local side-chain deformation. On the contrary, the FDCD spectrum when monitored at the monomer fluorescence region $(400-450 \mathrm{~nm})$ showed a smaller peaks than the absorption CD. Thus the conventional absorption $\mathrm{CD}$ represents an averaged $\mathrm{CD}$ for the helical and random portions of the polypeptide, as may be expected.

Summary of Spectroscopic Results and Discussion on the Electronic State of Poly[Asp $\left.\left(\mathrm{OCH}_{2}-9-A n t\right)\right]$

The spectroscopic findings are summarized as follows:

(1) Strong exciton couplet in CD spectrum indicates a helical arrangement of anthryl groups along a helical main chain.

(2) A presence of ground-state dimer or higher aggregates is evident from $\mathrm{CD}$ and absorption spectra.

(3) Fluorescence spectrum consisted mostly of monomer fluorescence with a small contribution from the dimer excited state or higher aggregates.

(4) CPF spectra suggested that the monomer excited state delocalizes over neighboring chromophores and that the dimer or aggregates has a dissymmetric structure. The excimer may be absent in this polymer.

(5) FDCD spectrum indicated that the dimer is formed in the helical part of the polymer.

The above observations fit to Scheme II.

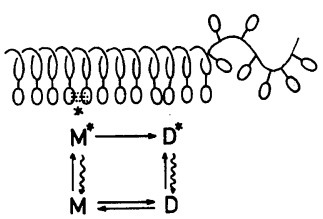

Scheme II

In the scheme the monomer excited state $\mathrm{M}^{*}$, in the helical part forms a partially delocalized exciton state and the energy migration among the exciton state is frequent. A part 
of the excited energy in the monomer excited state transfers to the dimer or higher aggregates $\mathrm{D}^{*}$, formed in the helical region. The excimer was neglected in this scheme. The larger $g_{\mathrm{ex}}$ than $g_{\mathrm{ab}}$ in FDCD spectrum indi cates that a randomly coiled portion is also present in this polymer. The content and the electronic states of the latter portion, however, cannot be studied by the chiroptical spectroscopy presented in this paper.

Acknowledgement. This work is partly supported by the Grant-in-Aid for Scientific Research (No. 59550626) from the Ministry of Education, Science, and Culture of Japan.

\section{REFERENCES}

1. Y. Wada, "Electronic Properties of Polymers," J. Mort and G. Pfister, Ed., John Wiley, N.Y., 1982, Chapter 4.

2. E. Fukada, Quart. Rev. Biophys., 16, 59 (1983).

3. D. J. Williams, Ed., "Nonlinear Optical Properties of Organic and Polymeric Materials," ACS Symposium Ser. 233, Washington D.C., 1983.

4. K. M. Ulmer, "Molecular Electronic Devices," F. L. Carter, Ed., Marcel Dekker, New York, N.Y., 1982,
Chapter 16.

5. M. Sisido, S. Egusa, and Y. Imanishi, J. Am. Chem. Soc., 105, 1041 (1983).

6. M. Sisido, S. Egusa, and Y. Imanishi, J. Am. Chem. Soc., 105, 4077 (1983).

7. S. Egusa, M. Sisido, and Y. Imanishi, Chem. Lett., 1307 (1983).

8. S. Egusa, M. Sisido, and Y. Imanishi, Macromolecules, 18, 882 (1985).

9. M. Sisido and Y. Imanishi, Macromolecules, 18, 890 (1985).

10. H. Nomori, M. Yoshikawa, and M. Hatano, Makromol. Chem., 177, 3077 (1976).

11. A. Ueno, T. Osa, and F. Toda, Macromolecules, 10, 130 (1977).

12. A. Ueno, F. Toda, and Y. Iwakura, Biopolymers, 13, 1213 (1974).

13. L. L. Chapoy, D. Biddle, J. Halstrom, K. Kovacs, K. Brunfeldt, M. A. Qasim, and T. Christensen, Macromolecules, 16, 181 (1983).

14. D. Biddle and L. L. Chapoy, Macromolecules, 17, 1751 (1984).

15. M. Sisido, S. Egusa, A. Okamoto, and Y. Imanishi, J. Am. Chem. Soc., 105, 3351 (1983).

16. E. W. Lobenstine, W. C. Schaefer, and D. H. Turner, J. Am. Chem. Soc., 103, 4936 (1981).

17. M. Sisido, "Kobunshi Jikkengaku 14, Seitai Kobunshi," Y. Imanishi and E. Tsuchida, Ed. (in Japanese), Kyoritsu Shuppan, Tokyo, 1984, pp 3654. 